\title{
Horizontal gene transfer increases microbiome stability
}

\author{
K. Z. Coyte ${ }^{1}$, C. Stevenson ${ }^{2}$, C. G. Knight ${ }^{3}$, E. Harrison ${ }^{2}$, J. P. J. Hall ${ }^{4}$, M. A. Brockhurst ${ }^{1}$
}

1. Division of Evolution and Genomic Sciences, Faculty of Biology, Medicine and Health, University of Manchester, Manchester M13 9PT, UK

2. Department of Animal and Plant Sciences, The University of Sheffield, Sheffield, S10 2TN, UK

3. School of Earth and Environmental Sciences, Faculty of Science and Engineering, University of Manchester, Manchester M13 9PT, UK

4. Department of Evolution, Ecology and Behaviour, Institute of Infection, Veterinary and Ecological Sciences, University of Liverpool, Liverpool, L69 7ZB, UK

Email for correspondence:

katharine.coyte@manchester.ac.uk, michael.brockhurst@manchester.ac.uk

\section{Abstract}

Genes encoding resistance to stressors, such as antibiotics or environmental pollutants, are widespread across microbiomes, often encoded on mobile genetic elements. Yet despite their prevalence, the impact of resistance genes and their mobility upon the dynamics of microbial communities remains largely unknown. Here we develop eco-evolutionary theory to explore how resistance genes alter the stability of diverse microbiomes. We show that adding resistance genes to a microbiome typically increases its overall stability, particularly for mobile resistance genes with high transfer rates that efficiently spread resistance throughout the community. However, the impact of resistance genes upon the stability of individual taxa varies depending upon the mobility of the resistance gene and the network of ecological interactions within the community. Nonmobile resistance genes can benefit susceptible taxa in cooperative communities, yet damage those in competitive communities. Moreover, whilst the transfer of mobile resistance genes generally increases the stability of previously susceptible recipient taxa to perturbation, it can, counterintuitively, decrease the stability of the originally resistant donor species. We confirmed these theoretical predictions experimentally using competitive soil microcosm communities. Here 
the stability of a susceptible microbial community to perturbation was increased by adding mobile resistance genes encoded on conjugative plasmids but was decreased when these same genes were encoded on the chromosome. Together these findings highlight the importance of horizontal gene transfer in driving the eco-evolutionary dynamics of diverse microbiomes.

\section{Background}

Diverse microbial communities colonize virtually every habitat on earth, shaping their abiotic environments and the health of their multicellular hosts ${ }^{1-3}$. Stably maintaining a diverse microbial community is critical for overall microbiome performance, ensuring that the presence of beneficial species or desirable metabolic traits are retained over time ${ }^{4-7}$. In particular, it is crucial that microbial communities can robustly withstand perturbations caused by external stressors, such

41 as environmental pollutants or antibiotics, which may otherwise dramatically reduce overall

42 microbiome abundances and diversity ${ }^{4,8-10}$. Antibiotic-induced changes in community composition

43 have been correlated with a range of adverse health outcomes in host-associated microbiomes ${ }^{11}$,

44 while losses in microbial diversity triggered by heavy-metal and other toxic pollution have been

45 linked to reduced nutrient cycling within environmental microbiomes ${ }^{12}$. Yet despite the importance

46 of withstanding perturbations, the forces shaping the stability of microbial communities remain

47 poorly understood.

49 Existing theoretical work on microbiome stability has focused primarily on the role of ecological

50 factors, developing mathematical models to disentangle how forces such as microbe-microbe

51 interactions or different classes of stressors influence how microbiomes respond to

52 perturbations ${ }^{13,14}$. However, such models have typically assumed that all species within a given

53 microbiome are equally affected by stressors. Perhaps more importantly, these models typically

54 also assume microbial species remain equally susceptible to stressors over time. In practice, 55 antibiotic or toxin resistance genes are prevalent within microbial communities, often encoded on 
56 mobile genetic elements such as plasmids or temperate phages, which can rapidly spread within

57 and between microbial species by horizontal gene transfer (HGT $)^{15-17}$. Therefore, not only are

58 species within microbiomes differentially impacted by stressors, but the rapid spread of mobile

59 genetic elements may dynamically alter the susceptibility of individual microbes to these stressors

60 over short periods of time. These resistance genes and their mobility are highly likely to influence

61 overall microbiome stability, yet exactly how remains unknown.

63 Here we develop eco-evolutionary theory to examine how the presence and mobility of resistance

64 genes within microbial communities shapes microbiome stability. We then test our key predictions

65 using model soil microbiomes exposed to heavy metal perturbations. In general, our modelling predicts that resistance genes increase overall microbiome stability, with this beneficial effect increasing with increasing gene mobility. However, we also find that a resistance gene can have very different impacts on differing community members, depending upon the precise balance of ecological interactions within a given microbiome, and the mobility of the resistance gene itself.

71 damage those in competitive communities. Meanwhile, though the spread of mobile resistance

72 genes tends to increase overall community stability, it can, counterintuitively, decrease the

73 stability of the originally resistant species. Crucially, our experiments support these key

74 predictions, confirming the beneficial impacts of resistance genes and their mobility on average

75 community properties, and recapitulating the adverse impacts of resistance genes on certain

76 community members. Overall, our work highlights the critical importance of eco-evolutionary

77 dynamics and horizontal gene transfer in shaping complex microbiomes.

79 Results and Discussion

80 Mathematical model of eco-evolutionary microbiome dynamics 
81 To understand the effect of resistance genes and their mobility on microbial community dynamics

82 we developed a simple and generalizable mathematical model of microbiome dynamics, built

83 around the generalized Lotka-Volterra (gLV) equations (Fig 1A). As in previous work ${ }^{18-22}$, our

84 model assumes the growth of each species within a microbiome is determined by the combination

85 of its own intrinsic growth rate $\left(r_{i}\right)$, its competition with kin $\left(s_{i}\right)$ and the combination of any

86 interactions each taxon has with other community members $\left(a_{i j}\right)$. Although simple, these gLV

87 models have been shown to well capture and predict the dynamics of both host-associated and

88 environmental microbial communities ${ }^{18-22}$. Now, we extended this basic model to explicitly

89 incorporate a stressor that inhibits (or kills) susceptible cells and a potentially mobile resistance

90 gene that protects cells encoding it, but at the cost of a reduced intrinsic growth rate. Using this

91 model, we could simulate the individual species abundances of any given microbiome over time.

92 More specifically, we could simulate the scenario in which a microbiome equilibrates, but is then

93 briefly exposed to an external stressor such as a heavy metal or an antibiotic perturbation (Fig

94 1B). By measuring the average change in species abundances during this perturbation we could

95 therefore quantify the stability of that microbiome.

97 Having established this basic model, we used it to explore the impact of resistance genes on the 98 stability of microbiome communities. Specifically, we generated a series of diverse multispecies 99 microbial communities, then quantified the stability of each of these microbiomes under four 100 distinct scenarios (Fig 1C). First, we simulated microbiome dynamics when all species were 101 susceptible to the stressor, and then again when a randomly chosen focal species carried a 102 resistance gene. Next, we repeated this process, but allowed each microbiome to initially 103 equilibrate in the presence of a low level of the stressor, for example, capturing prior exposure to 104 subinhibitory levels of antibiotics or pollutants. This process allows us to define two metrics: the 105 change in microbiome stability resulting from the introduction of a resistance gene, $\Delta \mathrm{R}$, and the 106 change in microbiome stability resulting from prior exposure to low-level selection, $\Delta \mathrm{E}$ (Fig 1D). 
107 For each community, we calculated $\Delta \mathrm{R}$ and $\Delta \mathrm{E}$ for the whole microbiome (Fig 1E), the focal

108 species only (i.e., the species that originally carried the resistance gene), and the background 109 community only (i.e., all species except the focal species). Using this modelling framework, we 110 could then explore in depth the impact of resistance genes on microbiome stability.

112 Mobile resistance genes increase stability of non-interacting communities

113 We began by exploring how the presence and mobility of resistance genes influenced the stability 114 of microbiomes in the absence of between-species ecological interactions (that is, all $a_{i j}=0$ ). To 115 do so, we generated a set of communities with and without resistance genes. We then 116 systematically varied the ability of these resistance genes to transfer within and between species 117 (Fig 2A), capturing all degrees of gene mobility from immobile (e.g., a chromosomally encoded 118 resistance gene) to highly mobile (e.g., a resistance gene encoded by a highly conjugative and 119 promiscuous plasmid).

121 Introducing any resistance gene increased overall microbiome stability, decreasing the average 122 drop in community abundances following the onset of the perturbation (Fig $2 \mathrm{~B}, \Delta \mathrm{R}>0$ ). However, 123 examining background and focal species separately revealed that in most cases this increase 124 was driven solely by the increased stability of the focal species. That is, the presence of a 125 resistance gene in the focal species drove up the average stability of the community as a whole, 126 but in most cases the stability of background species remained effectively unchanged (Fig 2B). 127 To substantially increase the stability of the background microbiome, we found that the novel 128 resistance gene must be highly mobile. This was because, prior to the perturbation, the resistance 129 gene did not confer any benefit, and thus could only spread into the background community when 130 its transfer rate exceeded its rate of decline caused by negative selection against its cost. 131 However, low-mobility resistance genes could increase background species stability provided 132 additional forces enhanced the spread of resistance prior to any perturbation. For example, prior 
133 exposure to weak stressor selection introduced a weak benefit to harboring the resistance gene

134 prior to the perturbation, enabling it to spread into the background community even at lower rates

135 of gene mobility (Fig 2C). As a consequence, prior exposure substantially increased the stability

136 of background species, even in communities harboring low-mobility resistance genes (Fig 2D).

\section{Interspecies interactions modulate the impact of resistance genes on microbiome stability}

139 Having established these baseline properties of the system, we next examined how the impact of 140 resistance genes upon community stability is modulated by interspecies interactions. Specifically,

141 we allowed individual species within our simulated communities to interact with one another in a 142 variety of different ways, ranging from competition and ammensalism (-/- and -/0 interactions 143 respectively), through exploitation (+/-), to cooperation and commensalism $(+/+$ and $+/ 0)$. We then 144 generated a range of different microbial communities, systematically varying the proportion of each interaction type (Fig 3A). As previously, we then simulated the introduction of resistance genes into these communities, also systematically changing the mobility of the resistance gene.

As in non-interacting communities, introducing any resistance gene typically increased overall community stability, and this increase was higher for more mobile resistance genes (Fig 3B, S15). However, this beneficial effect of resistance genes varied with interaction type and was far stronger in microbiomes with a high proportion of cooperative interactions. In these cooperative communities, individual species benefited both directly from acquiring resistance genes, and 153 indirectly from their cooperative partners acquiring resistance, which in turn helped to buffer the 154 negative impact of any perturbation. The principal effect of prior weak stressor exposure was once 155 again to reduce the level of gene mobility required for resistance genes to spread within the 156 community. And, as a consequence, this prior stressor exposure increased the stabilizing effect 157 of mobile resistance genes on overall community stability, across all interaction types (Fig 3E, S11585 , although, notably this effect was strongest for resistance genes with intermediate mobility). 
While mobile resistance genes were beneficial for overall microbiome stability, examining focal and background species separately revealed surprisingly different dynamics - with background and focal species showing markedly different responses to resistance genes depending upon the precise manner in which species were interacting and the mobility of the resistance gene (Fig 3C, D, S1-5). In cooperative microbiomes, background species benefited from the introduction of a resistance gene regardless of its mobility. This occurred because, by promoting the survival of species with whom a susceptible species cooperates, resistance genes aid recovery of the susceptible species regardless of whether they have access to the resistance gene through HGT. However, in competitive communities highly mobile resistance genes increased background community stability while low-mobility genes reduced background community stability (Fig 3C). That is, in highly competitive communities most species were less stable when another member

171 of the community harbored an immobile or low mobility resistance gene than when all species 172 were susceptible. What drove this phenomenon? In fully susceptible competitive communities, 173 during a perturbation every species experienced a reduction in their net growth rate, typically 174 resulting in a decrease in their overall abundance. However, as a consequence each species also 175 benefited from some competitive release - that is, the negative impact of competitors was 176 reduced as these competitors also decreased in abundance. In contrast, if one species acquired 177 an immobile or low-mobility resistance gene then this focal species remained at a high density 178 during the perturbation - and as such, susceptible species not only suffered from the stressor179 mediated inhibition, but also from continued strong competitive inhibition by the focal species.

181 This impact of competitive release also modulated the impact of prior selection - again with very 182 different impacts on background and focal species. Background species benefited from prior 183 exposure to low-level stressors regardless of community context (Fig 3F), as this initial selection 184 promoted the spread of mobile resistance into the community. Moreover, this spread of mobile 
resistance also stabilized cooperative focal species, as these species benefited from their cooperative partners acquiring resistance genes and thus remaining at high densities during perturbations (Fig 3G). However, in certain competitive communities prior selection could, counterintuitively, slightly reduce the stability of the focal species $(\Delta E<0$, Fig $3 G)$ because the spread of mobile resistance into background species meant that the focal species no longer benefited from any competitive release during perturbations. Altogether, our results suggest the introduction of a mobile resistance gene can have a wide variety of effects, with the exact impact depending upon which species are being examined, how they interact with one another, and the 193 mobility of the resistance gene.

\section{Experimental microbial communities reproduce theoretical predictions}

196 To test our predictions about the impact of resistance genes and their mobility on community 197 stability we developed an experimental model microbiome system. Specifically, we generated model microbiomes by inoculating sterile potting soil microcosms with 96 soil bacterial isolates

199 (our background community) and one focal species Pseudomonas fluorescens SBW25. In each microcosm this focal species was either susceptible to mercury $\left(\mathrm{Hg}^{\mathrm{S}}\right)$, or carried a mercury 201 resistance operon, encoded either on the chromosome or on one of two conjugative plasmids, 202 pQBR103 and pQBR57. Whereas the chromosomally encoded resistance is non-mobile, the 203 conjugative plasmids can transfer mercury resistance to other species ${ }^{23-25}$. Having assembled 204 these communities, we allowed them to equilibrate for ten serial transfers (40 days) either with or 205 without weak mercury selection (Fig 4A). After equilibrating, communities were perturbed with a 206 pulse of high concentration mercury, then propagated without mercury for two additional serial 207 transfers, mirroring the mode of perturbation used in our modelling framework. We determined 208 the composition of bacterial communities by 16S rDNA gene amplicon sequencing before and 209 after the perturbation, and estimated the abundances of the total community by colony counts. As 
210 in our models, we then quantified community stability as the change in species abundances

211 between the pre- and post-perturbation samples.

213 After equilibrating, each microbiome contained between three and five taxa at appreciable

214 abundances, namely: OTU_107 (the focal species, P. fluorescens SBW25), OTU_3

215 (Pseudomonas sp.), OTU_14 (Pseudomonas umsongensis), OTU_9 (Bacillus megaterium), and

216 OTU_19 (Bacillus simplex). Although the exact ecological interactions occurring between each of

217 these taxa are not known, there is good evidence for exploitative and interference competition

218 occurring between Pseudomonas species ${ }^{26,27}$, and between members of the Bacillus and

219 Pseudomonas genera ${ }^{28-31}$, suggesting this is likely to have been a competitive community. As

220 such, our model predicts that an introduced resistance gene would be likely to increase overall

221 microbiome stability, but that background species would only benefit from plasmid-encoded

222 resistance, and might suffer from the introduction of chromosomally encoded genes. Moreover,

223 our modelling also predicts that prior selection might, counterintuitively, slightly decrease the

224 stability of the focal species when it harbored plasmid-encoded mercury resistance, due to

225 transfer of mobile resistance limiting the benefits of competitive release.

227 As predicted, the presence of any resistance gene in the focal species SBW25 significantly 228 increased overall microbiome stability, strongly reducing the decline in abundance caused by the 229 mercury pulse (Fig 4C, effect of resistance $=0.030[0.025-0.035,95 \% \mathrm{Cl}]$, Bayesian linear model 230 BLM1, see SI). Similarly, as predicted, there was an overall positive effect on stability of prior 231 exposure to weak mercury selection (effect of exposure $=0.022[0.013-0.031,95 \% \mathrm{Cl}]$, BLM1).

232 We also observed a positive impact of gene mobility on overall microbiome stability, however, this 233 effect was small (estimated effect of mobility on stability, given resistance $=0.0065[0.0017-$ $2340.011,95 \% \mathrm{Cl}$ ], BLM1), and did not change significantly with prior exposure (estimated interaction 
effect between exposure and mobility, given resistance $=-0.0035[-0.014-0.007595 \% \mathrm{Cl}]$, BLM1) - reflecting that increases in stability were dominated by the behavior of the focal species.

238 In contrast, and as predicted by our mathematical modelling, calculating the stability of

239 background and focal species members separately revealed markedly different behavior between

240 species, and a far stronger effect of resistance gene mobility (Fig 4D). In communities without

241 prior selection, plasmid-encoded mobile resistance genes increased background community

242 stability relative to fully susceptible communities (estimated effect of resistance on stability, given

243 mobility $=0.015[0.010-0.020]$, Bayesian linear model BLM2, see SI). And, consistent with this

244 increased stability being driven by plasmid transfer into the background community, in these

245 communities we also observed significantly higher levels of mercury resistance in the background

246 species following the perturbation when plasmids were present (Fig 4D, effect of mobility on

247 resistance frequency, given resistance $=0.21[0.064-0.35]$ Bayesian linear model BLM3). In

248 contrast, however, adding immobile chromosomal resistance genes strongly reduced background

249 community stability (Fig. 4D, effect of chromosomal resistance $=-0.030[-0.036--0.025,95 \%$

$250 \mathrm{Cl}$, BLM 2). That is, in microbiomes harboring immobile resistance genes, background species

251 were more strongly perturbed by the mercury pulse than in microbiomes entirely lacking

252 resistance genes. Moreover, immobile resistance genes caused an even greater drop in

253 background species stability when compared to communities harboring mobile resistance genes

254 (effect of mobility on stability, given resistance $=0.046[0.041-0.050,95 \% \mathrm{Cl}], \mathrm{BLM} 2$ ). Together,

255 these results support our predictions that resistance genes can have markedly different impacts

256 on background community members, depending on their mobility.

258 Finally, we observed a striking difference in the response of the background and focal taxa to 259 prior mercury exposure. Specifically, when resistance was present within the community, prior 260 exposure increased the stability of the background species (effect of exposure given resistance 
$261=0.034[0.019-0.048,95 \% \mathrm{Cl}], \mathrm{BLM} 2)$ yet decreased the stability of the focal species

262 (interaction effect of focal community with exposure, given resistance $=-0.17[-0.19--0.14,95 \%$

$263 \mathrm{Cl}$, BLM2). And, notably, this decreased stability was unique to resistant focal species, with prior

264 exposure increasing the stability of the focal species if it was sensitive (interaction effect between

265 focal community and exposure $=0.12[0.10-0.14,95 \% \mathrm{Cl}]$, BLM2). This suggests that, as

266 predicted by our models, the increased stability of the background species might indeed be

267 reducing the competitive release experienced by the focal species during perturbations, and thus

268 decreasing the overall stability of the focal species following prior exposure (cf. red region in Fig

$2693 \mathrm{G}$ ). Collectively, these experimental findings support our theoretical predictions relating to the

270 effects of resistance genes and their mobility upon the stability of competition dominated

271 microbiomes.

273 Conclusions

274 Genes conferring resistance to stressors such as antibiotics, toxins or pollutants are widespread

275 within microbial communities, often encoded on mobile genetic elements such as plasmids or 276 temperate phages ${ }^{15-17}$. While the consequences of resistance - particularly to antibiotics - for

277 human health have been widely studied ${ }^{32}$, the impact that resistance genes have on the structure

278 and stability of microbial communities remains poorly understood. Here we combine novel theory

279 and experiments to disentangle the diverse ways in which resistance genes influence the stability

280 of microbiomes. Our work suggests that resistance genes typically increase overall community

281 stability, particularly when encoded on highly mobile genetic elements. However, exactly how

282 these genes influence microbiome properties depends upon the precise interplay between the

283 properties of the gene and of the underlying microbiome. The same gene may have directly

284 opposing effects upon microbiome stability, depending upon its mobility, or how individual species

285 interact with one another. Moreover, not all species are affected equally and, particularly in highly 
competitive microbiomes, the presence and transfer of resistance genes may benefit some species yet be detrimental to others.

The considerable variability in the effects of resistance genes within microbiomes introduces an

290 interesting set of potential conflicts between genes, their bacterial hosts, and the broader

291 ecosystem. For example, the spread of antimicrobial resistance poses a dangerous threat to

292 public health ${ }^{33}$. Yet within a given microbiome, the spread of resistance into susceptible taxa may

293 offer important ecological benefits, improving overall microbiome stability and protecting

294 susceptible community members from out-competition by resistant competitors. Increasing

295 community stability through the spread of mobile resistance genes could also enable the

296 maintenance of important ecosystem services within vulnerable microbial communities. Similarly,

297 transfer of a resistance gene into susceptible taxa may be advantageous for the fitness of the

298 individual gene, increasing its frequency within the community. However, in certain microbiomes

299 spread of the resistance gene may be costly for the original host - reducing its advantage over

300 otherwise susceptible competitors ${ }^{34}$. Together, these conflicts reveal the important and

301 sometimes complex effects mobile genetic elements can have upon microbial communities.

302 Moreover, they also underline the importance of considering exactly how properties such as

303 microbiome stability are quantified. Relying solely on coarse, whole-community metrics such as

304 overall community abundances or diversity may mask striking differences between individual

305 community members, and risks obscuring important eco-evolutionary dynamics.

307 To identify broad patterns in the impact of mobile resistance genes upon microbiomes here we

308 used relatively simple ecological models. The advantage of these simple models is that we can

309 analyze large numbers of microbiomes in high-throughput. However, as a consequence, there

310 are ecological and evolutionary features that we have not explicitly accounted for. For example,

311 previous studies have suggested that plasmid transfer is more likely between closer phylogenetic 
312 relatives, due to constraints on plasmid host-range or spatial structuring ${ }^{35}$. Indeed, in our

313 experiments plasmid-mediated transfer of $\mathrm{Hg}^{\mathrm{R}}$ from $P$. fluorescens SBW25 appeared to be limited

314 to congenerics - with more distantly related members of the community such as $B$. megaterium

315 apparently unable to gain the resistance genes, suggesting they were unable to acquire or

316 maintain either plasmid. Similarly, our model does not explicitly include de novo mutations or

317 phenotypic changes that may modulate how individual taxa respond to stressors without requiring

318 the acquisition of resistance genes from other community members. Indeed, in our experiments

319 we observed an increase in the stability of background species after prior exposure to mercury

320 within the chromosomally-encoded resistance treatment (Fig. 4D), suggesting mechanisms other

321 than plasmid transfer may have driven this increased stability ${ }^{36}$. Each of these phenomena has

322 the potential to further modulate the impact of horizontal gene transfer upon microbiome stability,

323 and disentangling exactly how will be critical if we wish to fully understand and ultimately predict

324 microbiome dynamics.

\section{Materials and Methods}

\section{Underlying microbiome model}

329 In line with previous work ${ }^{18,19}$, we model each microbiome as a network, where each node 330 represents a species and each edge captures the interaction between them. However, now we 331 extend this model to include two populations for each species, one with plasmids and one

332 without ${ }^{25}$. For each species $i$, plasmid-free cells grow at a rate $r_{i}$ and plasmid-bearing cells grow 333 at a rate $r_{i}-c$, where $c$ indicates the cost of plasmid carriage. Plasmids can transfer within and 334 between species, with the per cell rate of plasmid transfer from species $j$ into species $i$ defined as $335 \gamma_{i j}=\bar{\gamma}+\epsilon_{i j}$, where $\bar{\gamma}$ is the average plasmid transfer rate, and $\epsilon_{i j}$ is a noise term drawn from a 336 Normal distribution to introduce variability in plasmid transfer rates between species (note, in 
instances where $\gamma_{i j}<0$ we set $\gamma_{i j}=0$ ). Finally, we introduce an inhibition term $-\beta D$, where D

338 defines the level of antibiotic or toxin in the environment, and $\beta$ the susceptibility of the population

339 in question. More specifically, we set $\beta^{s}=1$ for the susceptible population, and $\beta^{r}=0.1$ for the

340 resistant population. Together, this enables us to define the growth rate of the plasmid-negative,

$341 X^{s}$, and plasmid-positive, $X^{r}$, populations of a given species as,

$$
\frac{d X_{i}^{s}}{d t}=X_{i}^{s}\left(r_{i}-s_{i}\left(X_{i}^{s}+X_{i}^{r}\right)+\sum_{j=1, j \neq i}^{N} a_{i j} X_{j}^{s}+\sum_{j=1, j \neq i}^{N} a_{i j} X_{j}^{r}\right)-\sum_{j=1}^{N} \gamma_{i j} X_{i}^{s} X_{j}^{r}-\beta^{s} D X_{i}^{s}
$$

$$
\frac{d X_{i}^{r}}{d t}=X_{i}^{r}\left(\left(r_{i}-c\right)-s_{i}\left(X_{i}^{s}+X_{i}^{r}\right)+\sum_{j=1, j \neq i}^{N} a_{i j} X_{j}^{s}+\sum_{j=1, j \neq i}^{N} a_{i j} X_{j}^{r}\right)+\sum_{j=1}^{N} \gamma_{i j} X_{i}^{s} X_{j}^{r}-\beta^{r} D X_{i}^{r}
$$

with equivalent expressions defining the dynamics of each other species $j=2: N$ within the results (Fig S5).

Having established this new model, we generated a series of microbiomes, each composed of $N=10$ species. Within any given microbiome, each species $\mathrm{i}$ interacts with species $\mathrm{j}$ with probability C. To investigate how interspecies interactions modulate the effect of resistance genes, we

354 systematically alter the proportion of individual interaction terms, $a_{i j}$, that are positive, $\mathrm{P}_{\mathrm{m}}$, such 355 that when $\mathrm{P}_{\mathrm{m}}=0$ the community is purely competitive, when $\mathrm{P}_{\mathrm{m}}=0.5$ the community contains 356 a mixture of all interaction types, from competitive and ammensal, through exploitation, to cooperative and commensal, and when $\mathrm{P}_{\mathrm{m}}=1$ the community is purely cooperative. Finally, we choose the magnitude of each $a_{i j}$ is from a half-normal distribution with standard deviation $\sigma=$ 
stable equilibrium $X_{i}^{*} \approx 1 \forall i \in 1, \ldots, N$ in the absence of any resistance gene. Importantly, we find similar results when varying each of these key microbiome parameters (Figs S3-5).

\section{Quantifying microbiome stability}

364 We calculate the stability of any given community by simulating its dynamics in response to a 365 perturbation. Specifically, we allow the community to equilibrate, then briefly perturb it by setting 366 the stressor level within the environment to $D=0.1$ for $t=25$ time units. We then measure the 367 difference in abundance of each species $i$ before, $A_{i}^{b}$, and after, $A_{i}^{a}$, this perturbation. We define 368 the stability of each individual species $i$ based on their fold change in response to the perturbation,

369 such that Stability $_{i}=\log _{10} \frac{A_{i}^{a}}{A_{i}^{b}}$, then define the stability of the whole community (or background 370 community), as the mean of Stability $_{i}$ across all species (or across only the background 371 species). To explore the impact of resistance genes on stability for each community we perform

372 these simulations when all species are susceptible to the stressor, and when one randomly 373 chosen species harbors a mobile resistance gene. To explore the impact of low-level selection on 374 stability, we perform these simulations when the community initially equilibrates in a stressor-free 375 environment, and when the community equilibrates in the presence of a low-level of the stressor 376 (setting $D=0.01$ during the equilibration period), with all simulations performed in MATLAB 377 R2020a.

\section{Strains and culture conditions}

380 To test the accuracy of our predictions, we assembled experimental model microbiomes, 381 composed of a defined background community augmented with a predetermined focal species.

382 For our focal species we used Pseudomonas fluorescens SBW25 labelled with a gentamicin 383 resistance marker using the mini-Tn7 transposon system ${ }^{25,36-38}$. More specifically, we generated 384 independent $P$. fluorescens strains that were either susceptible to mercury, or harbored harboured 
a mercury resistance gene, $\mathrm{Hg}^{\mathrm{R}}$, on either the chromosome, the conjugative plasmid $\mathrm{pQBR} 57^{38,39}$, or the conjugative plasmid $\mathrm{PQBR} 103^{39}$. Individual colonies of each strain (one for each replicate) were isolated on selective KB agar and grown overnight at 28 degrees in $6 \mathrm{ml} \mathrm{KB}$ broth (10 g glycerol, $20 \mathrm{~g}$ proteose peptone no. $3,1.5 \mathrm{~g} \mathrm{~K}_{2} \mathrm{HPO}_{4} \cdot 3 \mathrm{H}_{2} \mathrm{O}, 1.5 \mathrm{~g} \mathrm{MgSO}_{4} \cdot 7 \mathrm{H}_{2} \mathrm{O}$, per litre ${ }^{40}$ ) in a shaking incubator.

To generate our background community, we plated supernatant from non-autoclaved John Innes No.2 potting soil on nutrient agar plates, which were then grown for 48 hours in a 28 degree centigrade incubator. Following this incubation period, we randomly selected 96 colonies, which we screened against $\mathrm{Hg}^{2+}$ and $\mathrm{Gm}$ to ensure no community members already harbored phenotypic resistance to either our stressor or selective marker. Each of these bacterial strains were grown separately overnight in $6 \mathrm{ml} \mathrm{KB}$ broth in a shaking incubator and then washed and mixed at an equal volume to make the background community. To generate each final microbiome and used $100 \mu$ to initiate each experimental replicate. Populations were cultured in sterile soil microcosms consisting of 10 grams twice autoclaved John Innes No.2 potting soil supplemented with $900 \mu \mathrm{l}$ of sterile $\mathrm{H}_{2} \mathrm{O}$ and maintained at 28 degrees at $80 \%$ relative humidity.

\section{Community perturbation experiment}

405 We established twelve replicate communities per SBW25 genotype: SBW25, SBW25 with 406 chromosomal $\mathrm{Hg}^{\mathrm{R}}$, SBW25 carrying $\mathrm{Hg}^{\mathrm{R}}$ encoded on PQBR57, SBW25 carrying $\mathrm{Hg}^{\mathrm{R}}$ encoded on 407 pQBR103. These were propagated by serial transfer of $1 \%$ of the community every 4 days into

408 fresh soil microcosms for twelve transfers. In half of these lines communities were augmented 409 with mercuric chloride (at $16 \mu \mathrm{g} / \mathrm{g} \mathrm{HgCl}_{2}$ ) from transfer 2 onwards, allowing us to capture 410 communities with and without prior stressor exposure. At transfer 10 all communities were 
411 perturbed by exposure to $128 \mu \mathrm{g} / \mathrm{g} \mathrm{HgCl}_{2}$ and then propagated for a further serial transfer at 0

$412 \mu \mathrm{g} / \mathrm{g} \mathrm{HgCl}_{2}$. Samples of each community were cryogenically stored at each serial transfer in $20 \%$

413 glycerol. Throughout the experiment, we determined the population density of SBW25 by diluting

414 and plating samples onto KB agar supplemented with $6 \mu \mathrm{g} / \mathrm{ml}$ gentamicin, and the abundance of

415 the entire community by plating onto nutrient agar. In addition, we determined the frequency of

416 the $\mathrm{Hg}^{\mathrm{R}}$ phenotype in the community as a whole by plating onto nutrient agar supplemented with

$41720 \mu \mathrm{M} \mathrm{HgCl}_{2}$, while we determined the frequency of $\mathrm{Hg}^{\mathrm{R}}$ resistance in the focal strain by plating

418 onto nutrient agar supplemented with $20 \mu \mathrm{M} \mathrm{HgCl} 2$ and $6 \mu \mathrm{g} / \mathrm{ml}$ gentamicin.

\section{$16 S$ rRNA gene sequencing and analysis}

421 To quantify community composition we extracted whole community DNA samples from the

422 thawed stocks stored on days 10 and 11 (i.e. before and after the mercury shock, Fig 4a).

423 Specifically, we extracted DNA using QIAGEN DNeasy PowerSoil kits, following manufacturer

424 instructions with the exception that stocks were initially spun down and re-suspended in 1x M9 to

425 remove glycerol. DNA concentrations were assessed using Qubit fluorometer 3.0 (Thermo Fisher

426 Scientific) and diluted to $20 \mathrm{ng} \mathrm{\mu l}^{-1}$ where possible before samples were sent for downstream PCR

427 amplification of the v4 region of 16s rRNA gene and sequencing on the Illumina MiSeq platform.

428 The raw forward and reverse reads were merged and processed using QIIME ${ }^{41}$. Reads were

429 stripped of their primer and barcoding sequences using Cutadapt ${ }^{42}$ and untrimmed reads were

430 discarded. Reads were truncated at 254 bp (size of the amplicon). Reads were then dereplicated

431 using Vsearch ${ }^{43}$ and clustered into operational taxonomic units (OTUs) using Usearch ${ }^{44}$ with $97 \%$

432 similarity. OTUs were then filtered based on OTUs which appeared in the positive control (14

433 OTUs in total)._Putative taxonomic identification of the 14 OTU's was performed using BLAST ${ }^{45}$

434 to align the OTU sequence data to the NCBI nucleotide database, listed in SI Table 2. Total

435 abundances of the focal species and the background community were determined by multiplying

436 the relative abundances of each with the total community abundances calculated by CFU counts. 


\section{Statistics}

439 Differences in microbiome stability and resistance frequency were estimated using three Bayesian

440 linear models, accounting for the experimental structure (where relevant, the presence of

441 resistance, its mobility, foreground versus background communities, prior exposure to weak

442 mercury selection and timing of measurement relative to mercury shock), non-homogeneity of

443 variances and, where appropriate, non-normality of residuals, using broad priors. These models

444 were fitted with the brms package ${ }^{46}$ (version 2.16.3) which uses STAN via the $R$ language ${ }^{47}$

445 (version 4.1.2). Four MCMC chains were used, each of 4,000 iterations, where the first 2,000

446 were discarded as warm-up, resulting in 8,000 draws from the posterior distribution. Convergence

447 was checked visually using plots of the draws and via the R-hat value ${ }^{48}$, which will equal 1 at

448 convergence and was 1.0 for all parameter estimates reported in the main text and Supplemental

449 Information. All values are reported as a mean with $95 \%$ credibility interval $(\mathrm{Cl})$. Details of model

450 structures and estimated parameters for each of the three models (Supplemental Tables 3-5) are

451 given in the Supplemental Information.

453 Acknowledgements

454 We thank D.R. Gifford and W.P.J. Smith for helpful discussions. K.Z.C. was funded by a University 455 of Manchester Presidential Fellowship. C.S. was supported by an ACCE DTP NERC PhD 456 studentship. M.A.B., J.P.J.H. and E.H. were funded by grants BB/R014884/1 and NE/R008825/1.

\section{Competing interests}

459 We declare no competing interests. 
463 1. Whitman, W. B., Coleman, D. C. \& Wiebe, W. J. Prokaryotes: the unseen majority. Proc.

$464 \quad$ Natl. Acad. Sci. U. S. A. 95, 6578-83 (1998).

465 2. Lynch, S. V. \& Pedersen, O. The Human Intestinal Microbiome in Health and Disease. N.

$466 \quad$ Engl. J. Med. 375, 2369-2379 (2016).

467 3. Van Der Heijden, M. G. A., Bardgett, R. D. \& Van Straalen, N. M. The unseen majority:

468 soil microbes as drivers of plant diversity and productivity in terrestrial ecosystems. Ecol.

$469 \quad$ Lett. 11, 296-310 (2008).

4704 4. Dethlefsen, L. \& Relman, D. A. Incomplete recovery and individualized responses of the 471 human distal gut microbiota to repeated antibiotic perturbation. Proc. Natl. Acad. Sci. U.

472 S. A. 108 Suppl 1, 4554-61 (2011).

473 5. Theriot, C. M., Bowman, A. A. \& Young, V. B. Antibiotic-Induced Alterations of the Gut 474 Microbiota Alter Secondary Bile Acid Production and Allow for Clostridium difficile Spore 475 Germination and Outgrowth in the Large Intestine. mSphere 1, (2016).

476 6. Allison, S. D. \& Martiny, J. B. H. Resistance, resilience, and redundancy in microbial 477 communities. Proc. Natl. Acad. Sci. 105, 11512-11519 (2008).

478 7. Bell, T., Newman, J. A., Silverman, B. W., Turner, S. L. \& Lilley, A. K. The contribution of 479 species richness and composition to bacterial services. Nat. 2005 4367054 436, 1157$4801160(2005)$.

481 8. Liu, Y. R., Delgado-Baquerizo, M., Bi, L., Zhu, J. \& He, J. Z. Consistent responses of soil microbial taxonomic and functional attributes to mercury pollution across China.

484 9. Griffiths, B. S. \& Philippot, L. Insights into the resistance and resilience of the soil 485 microbial community. FEMS Microbiol. Rev. 37, 112-129 (2013).

486 10. Schwartz, D. J., Langdon, A. E. \& Dantas, G. Understanding the impact of antibiotic 487 perturbation on the human microbiome. Genome Med. 12, 1-12 (2020).

488 11. Taur, Y. et al. Intestinal domination and the risk of bacteremia in patients undergoing 
allogeneic hematopoietic stem cell transplantation. Clin. Infect. Dis. 55, 905-14 (2012).

12. Kandeler, E., Kampichler, C. \& Horak, O. Influence of heavy metals on the functional diversity of soil microbial communities. Biol. Fertil. Soils 1996233 23, 299-306 (1996).

13. Coyte, K. Z., Schluter, J. \& Foster, K. R. The ecology of the microbiome: Networks, competition, and stability. Science (80-. ). 350, (2015).

14. Shaw, L. P. et al. Modelling microbiome recovery after antibiotics using a stability landscape framework. ISME J. 13, 1845-1856 (2019).

15. Allen, H. K. et al. Call of the wild: antibiotic resistance genes in natural environments. Nat. Rev. Microbiol. 201084 8, 251-259 (2010).

16. Mclnnes, R. S., McCallum, G. E., Lamberte, L. E. \& van Schaik, W. Horizontal transfer of antibiotic resistance genes in the human gut microbiome. Curr. Opin. Microbiol. 53, 3543 (2020).

17. Silver, S. \& Misra, T. K. PLASMID-MEDIATED HEAVY METilL RESISTANCES. Ann.

503 18. Coyte, K. Z., Schluter, J. \& Foster, K. R. The ecology of the microbiome: Networks, competition, and stability. Science (80-. ). 350, 663-666 (2015).

19. Coyte, K. Z., Rao, C., Rakoff-Nahoum, S. \& Foster, K. R. Ecological rules for the assembly of microbiome communities. PLOS Biol. 19, e3001116 (2021).

20. Mounier, J. et al. Microbial interactions within a cheese microbial community. Appl. Environ. Microbiol. 74, 172-181 (2008).

509 21. Marino, S., Baxter, N. T., Huffnagle, G. B., Petrosino, J. F. \& Schloss, P. D. Mathematical modeling of primary succession of murine intestinal microbiota. Proc. Natl. Acad. Sci. U.

512 22. Thingstad, T. F. Elements of a theory for the mechanisms controlling abundance, 513 diversity, and biogeochemical role of lytic bacterial viruses in aquatic systems. Limnol.

$514 \quad$ Oceanogr. 45, 1320-1328 (2000). 
23. Kottara, A., Hall, J. P. J., Harrison, E. \& Brockhurst, M. A. Variable plasmid fitness effects and mobile genetic element dynamics across Pseudomonas species. FEMS Microbiol. Ecol. 94, 172 (2018).

24. Kottara, A., Hall, J. P. J. \& Brockhurst, M. A. The proficiency of the original host species determines community-level plasmid dynamics. FEMS Microbiol. Ecol. 97, (2021).

25. Hall, J. P. J., Wood, A. J., Harrison, E. \& Brockhurst, M. A. Source-sink plasmid transfer dynamics maintain gene mobility in soil bacterial communities. Proc. Natl. Acad. Sci. 113,

26. Jousset, A., Eisenhauer, N., Merker, M., Mouquet, N. \& Scheu, S. High functional diversity stimulates diversification in experimental microbial communities. Sci. Adv. 2, (2016).

27. Hall, J. P. J., Harrison, E. \& Brockhurst, M. A. Competitive species interactions constrain abiotic adaptation in a bacterial soil community. Evol. Lett. 2, 580-589 (2018).

28. Simões, M., Simoes, L. C., Pereira, M. O. \& Vieira, M. J. Antagonism between Bacillus cereus and Pseudomonas fluorescens in planktonic systems and in biofilms. https://doi.org/10.1080/08927010802239154 24, 339-349 (2008).

29. Garbeva, P., Silby, M. W., Raaijmakers, J. M., Levy, S. B. \& Boer, W. De. Transcriptional and antagonistic responses of Pseudomonas fluorescens Pf0-1 to phylogenetically different bacterial competitors. ISME J. 201156 5, 973-985 (2011).

534 30. Powers, M. J., Sanabria-Valentín, E., Bowers, A. A. \& Shank, E. A. Inhibition of cell differentiation in Bacillus subtilis by Pseudomonas protegens. J. Bacteriol. 197, 2129_

537 31. Molina-Santiago, C. et al. The extracellular matrix protects Bacillus subtilis colonies from

540 32. Friedman, N. D., Temkin, E. \& Carmeli, Y. The negative impact of antibiotic resistance. 
Clin. Microbiol. Infect. 22, 416-422 (2016).

542 33. No time to Wait: Securing the future from drug-resistant infections. Available at: https://www.who.int/publications/i/item/no-time-to-wait-securing-the-future-from-drugresistant-infections. (Accessed: 5th January 2022)

34. Eberhard, W. G. Evolution in bacterial plasmids and levels of selection. Q. Rev. Biol. 65, 3-22 (1990).

35. Thomas, C. M. \& Nielsen, K. M. Mechanisms of, and Barriers to, Horizontal Gene Transfer between Bacteria. Nat. Rev. Microbiol. 200539 3, 711-721 (2005).

36. Harrison, E., Hall, J. P. J., Paterson, S., Spiers, A. J. \& Brockhurst, M. A. Conflicting selection alters the trajectory of molecular evolution in a tripartite bacteria-plasmidphage interaction. Mol. Ecol. 26, 2757-2764 (2017).

37. Lambertsen, L., Sternberg, C. \& Molin, S. Mini-Tn7 transposons for site-specific tagging of bacteria with fluorescent proteins. Environ. Microbiol. 6, 726-732 (2004).

554 38. Stevenson, C., Hall, J. P. J., Harrison, E., Wood, A. J. \& Brockhurst, M. A. Gene mobility promotes the spread of resistance in bacterial populations. ISME J. 201711811,1930 1932 (2017).

39. Hall, J. P. J. et al. Environmentally co-occurring mercury resistance plasmids are

40. King, E. O., Ward, M. K. \& Raney, D. E. Two simple media for the demonstration of pyocyanin and fluorescin. J. Lab. Clin. Med. 44, 301-307 (1954).

41. Caporaso, J. G. et al. QIIME allows analysis of high-throughput community sequencing

564 42. Martin, M. Cutadapt removes adapter sequences from high-throughput sequencing reads. EMBnet.journal 17, 10-12 (2011).

566 43. Rognes, T., Flouri, T., Nichols, B., Quince, C. \& Mahé, F. VSEARCH: A versatile open 
source tool for metagenomics. PeerJ 2016, e2584 (2016).

568 44. Edgar, R. C. \& Bateman, A. Search and clustering orders of magnitude faster than BLAST. Bioinformatics 26, 2460-2461 (2010).

570 45. Altschul, S. F., Gish, W., Miller, W., Myers, E. W. \& Lipman, D. J. Basic local alignment 571 search tool. J. Mol. Biol. 215, 403-410 (1990).

572 46. Bürkner, P. C. brms: An R Package for Bayesian Multilevel Models Using Stan. J. Stat.

$573 \quad$ Softw. 80, 1-28 (2017).

574 47. The R Foundation. R: The R Project for Statistical Computing. (2018). Available at:

575 https://www.r-project.org/. (Accessed: 11th February 2022)

576 48. Vehtarh, A., Gelman, A., Simpson, D., Carpenter, B. \& Burkner, P. C. Ranknormalization, folding, and localization: an improved $\mathrm{R}$ for assessing convergence of MCMC (with discussion). Bayesian Anal. 16, 667-718 (2021). 
A

C
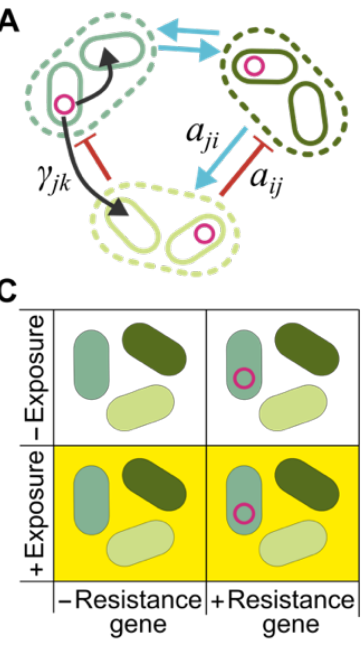

E

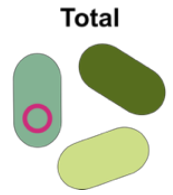

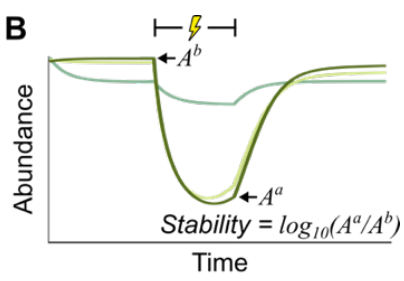

D

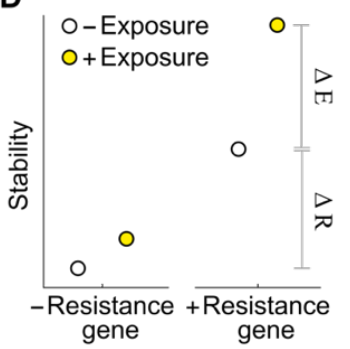

Figure 1. Mathematical modelling captures eco-evolutionary dynamics of microbial

communities. A. Schematic illustrating our mathematical model; each species (dashed line) is

584 composed of two populations, with and without a resistant gene. Species impact one another's

585 growth through ecological interactions such as competition or competition (blue and red arrows),

586 while horizontal gene transfer enables resistance genes to spread within and between species

587 (black arrows). B. Schematic illustrating representative microbiome dynamics, capturing species

588 dynamics before, during, and after an external perturbation (lightning bolt). We calculate each

589 species's abundance immediately before, $A^{b}$, and after, $A^{a}$, the perturbation period, then define

590 stability as the average logged fold change for each species (mean $\left.\log _{10}\left(A^{a} / A^{b}\right)\right)$ C. Schematic

591 illustrating our four modelling scenarios: communities with and without resistance genes, and with

592 and without prior exposure to low-level stressors. D. Comparing the four scenarios allows us to

593 calculate the change in microbiome stability that results from the introduction of a resistance gene

$594(\Delta R)$, and the change in stability that results from prior exposure to weak low-level selection $(\Delta \mathrm{E})$.

595 E. To disentangle the impact of resistance and selection on different taxa we calculate $\Delta R$ and

$596 \Delta \mathrm{E}$ for the total community, the background community only and the focal species alone. 
A Susceptible

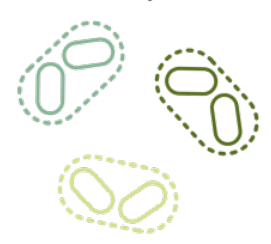

B

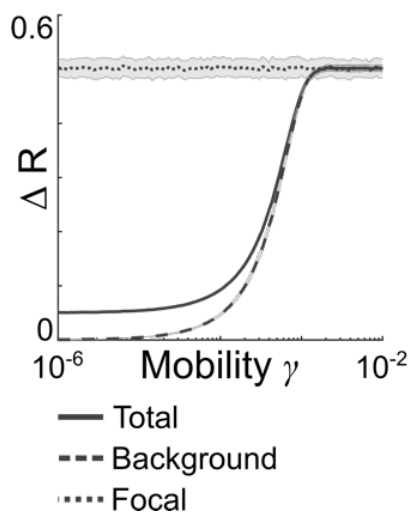

Mobility: $\gamma$

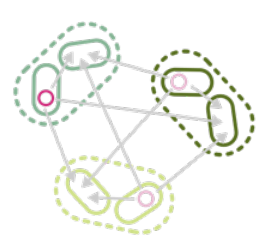

C

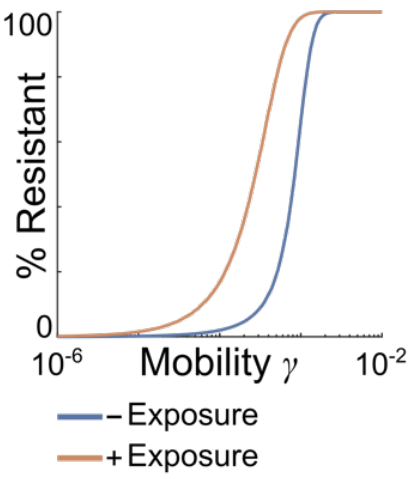

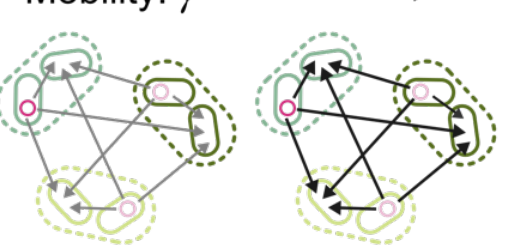

D

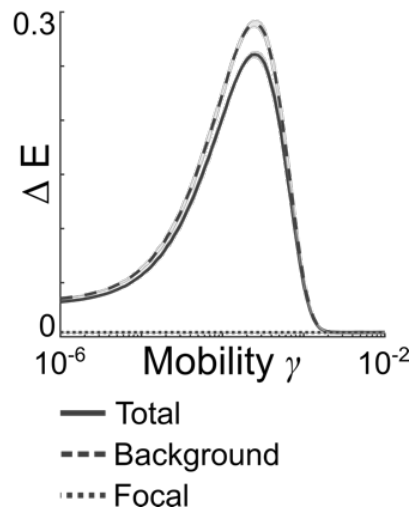

598 Figure 2. In simple communities mobile resistance genes increase microbiome stability.

599 A. Schematic illustrating our modelling approach. We generate a set of simple microbiomes

600 without between species interactions, then for each community we simulate the introduction of a

601 series of resistance genes with increasing mobility. B. $\Delta R$, the change in stability resulting from

602 the introduction of a resistance gene, for the community as a whole (solid line), the background

603 community (dashed line) and the focal species (dotted line). Any resistance gene significantly

604 increases overall community stability, but only highly mobile resistance genes substantially

605 increase the stability of background species. C. Plasmid frequency within the background

606 community with (orange) and without (blue) prior exposure to low-level stressors. Prior exposure

607 increases plasmid frequency, with this increase greatest for plasmids with intermediate mobility.

608 D. $\Delta \mathrm{E}$, the change in stability resulting from prior exposure to low stressor levels, for the

609 community as a whole (solid line), the background community (dashed line) and the focal species

610 (dotted lined). Prior low-level selection increases the stability of both the community as a whole

611 and background species, with this effect greatest for communities with intermediate mobility

612 plasmids. Throughout lines and error bars represent mean and standard deviation over 100

613 independent, 10-species communities, with model parameters given in Table 1. 

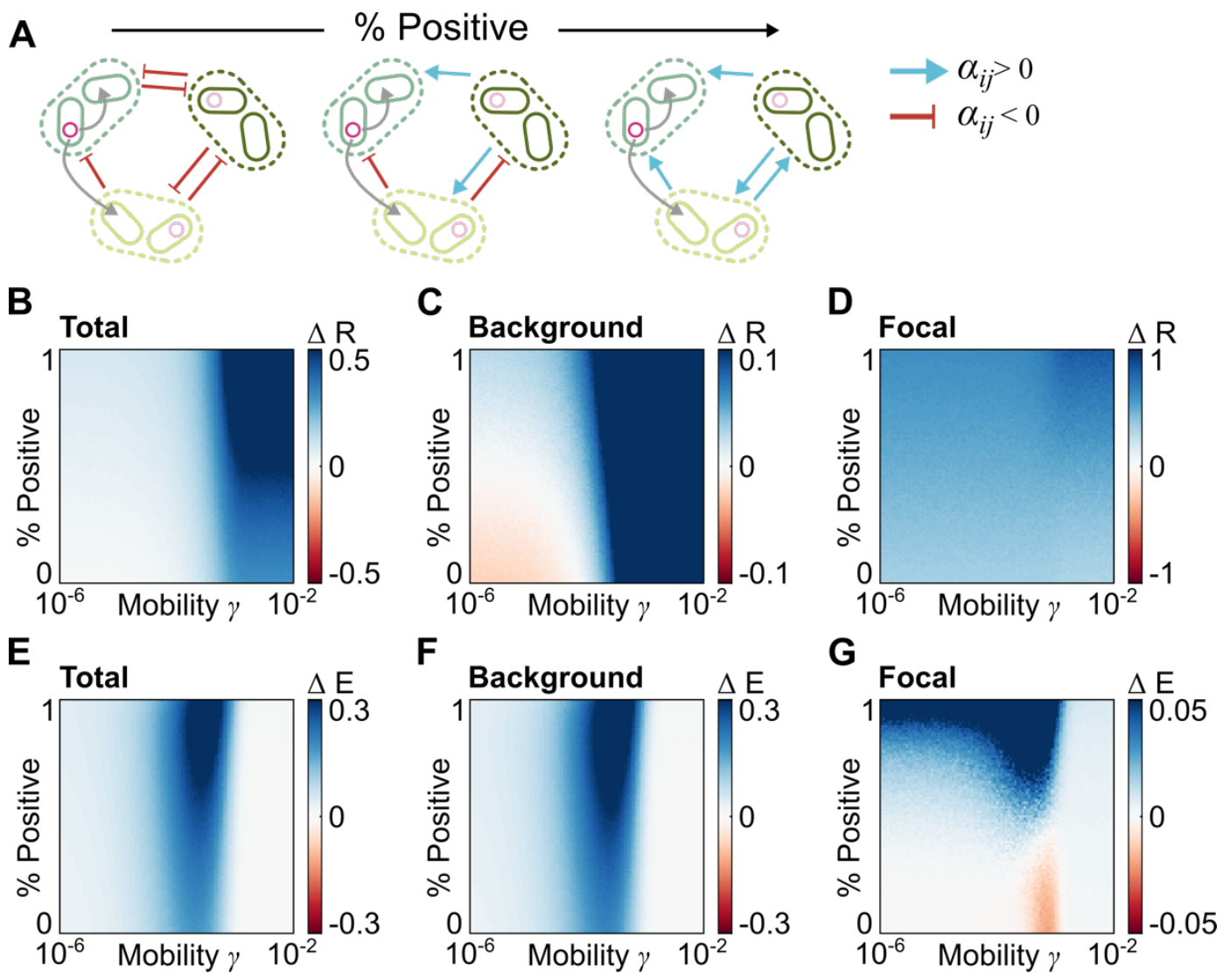

615 Figure 3. Interspecies interactions modulate the effect of resistance genes. A. Schematic

616 illustrating our modelling approach. We generated a series of diverse microbiomes, systematically

617 increasing the frequency with which microbes facilitate one another's growth. B-D. Average $\Delta R$,

618 the change in stability resulting from the introduction of a resistance gene, under varying

619 community types and plasmid transfer rates, shown for the whole microbiome $(\mathrm{B})$, the background

620 community (C) and the focal species alone (D). E-G. Average $\Delta \mathrm{E}$, the change in stability resulting

621 from prior exposure to low stressor levels, under varying community types and plasmid transfer

622 rates, shown for the whole microbiome $(E)$, the background community $(F)$ and the focal species

623 alone (G). Throughout patch color represents mean $\Delta \mathrm{R}$ or $\Delta \mathrm{E}$ over 100 independent, 10-species

624 communities, across a range of 101 Positivity and $\gamma$ values. Other model parameters given in 625 Table 1. 
A

16S rDNA sequencing $\mathbb{I} \mathbb{N} \mathbb{N}$

CFU counts

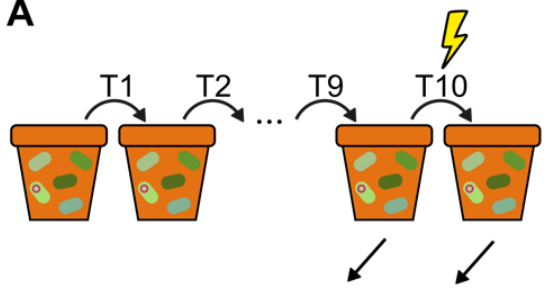

\section{每}
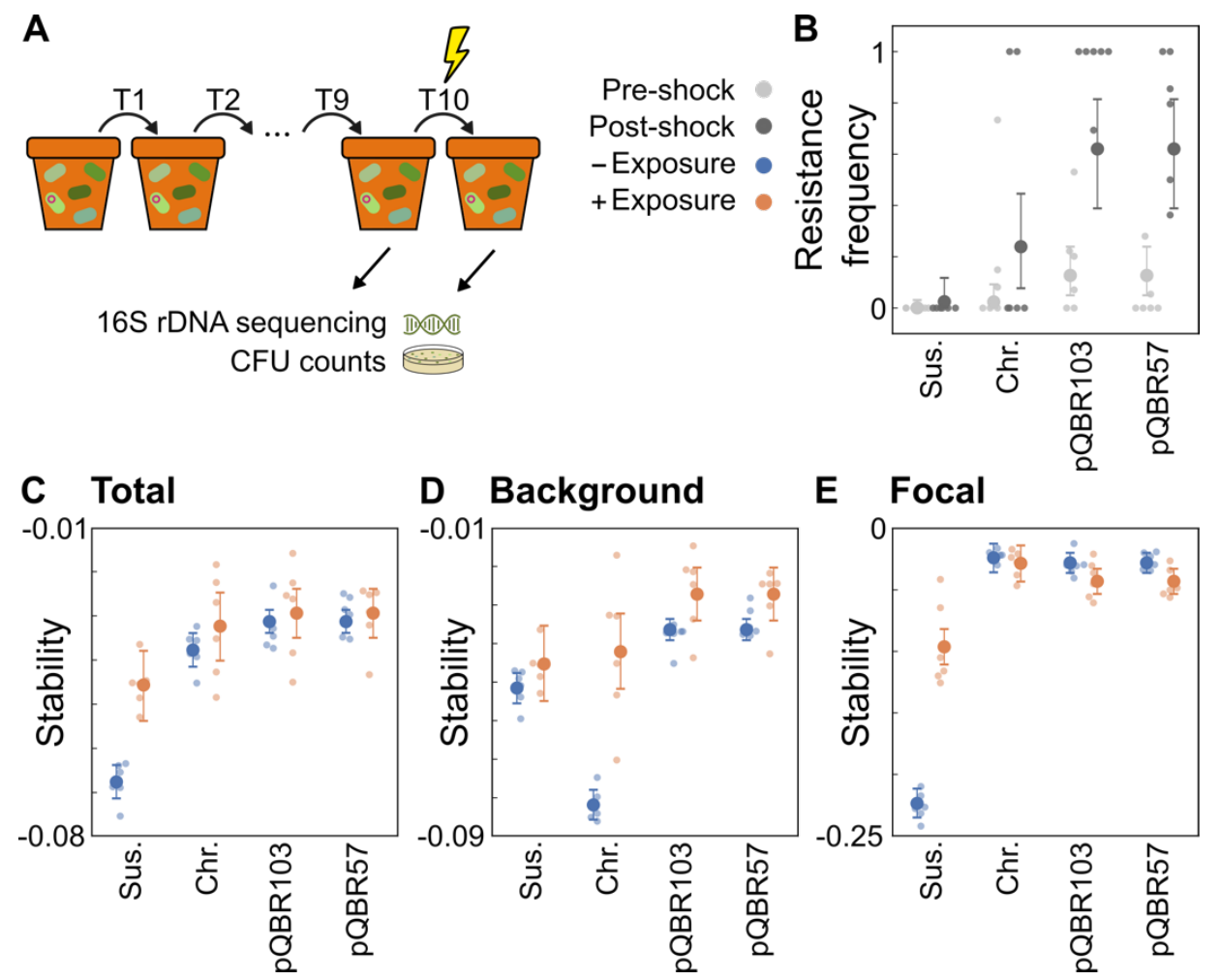

D Background

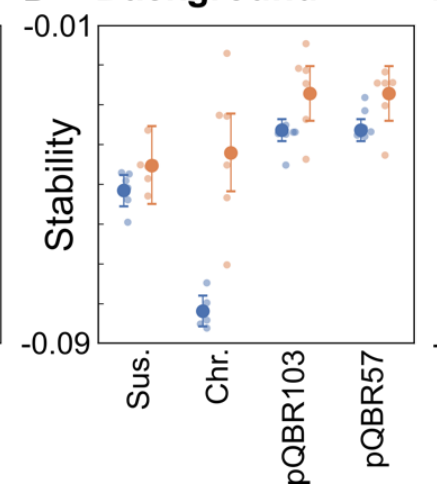

E Focal

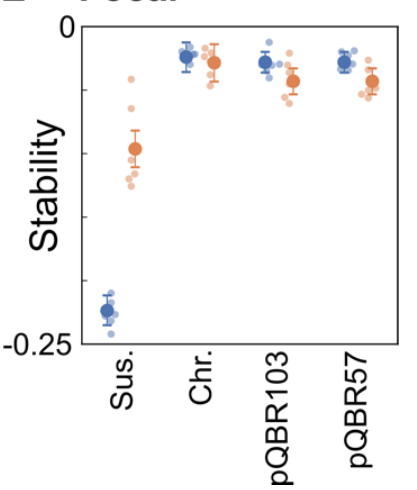

Figure 4. Experimental microbiomes recapitulate the predicted impact of resistance genes

631 level mercury, then perturbed with a high-level mercury pulse. B. Frequency of resistance within

632 the background population before (light grey) and after (dark grey) the high-level mercury pulse,

633 calculated for each experimental condition (fully susceptible, Sus., and Chromosomal, Chr. or

634 plasmid-carried resistance, pQBR103, pQBR57) C. Total microbiome stability, as measured by

635 log10(Fold Change) in species abundances following perturbation, such that more negative

636 values indicate a less stable community. D. Stability of the background community individually. E.

637 Stability of the focal species alone. Throughout, orange and blue dots indicate community stability

638 with and without prior mercury exposure respectively, with each condition (resistance $\mathrm{x}$ prior

639 selection) containing $n=6$ independent samples. 
A
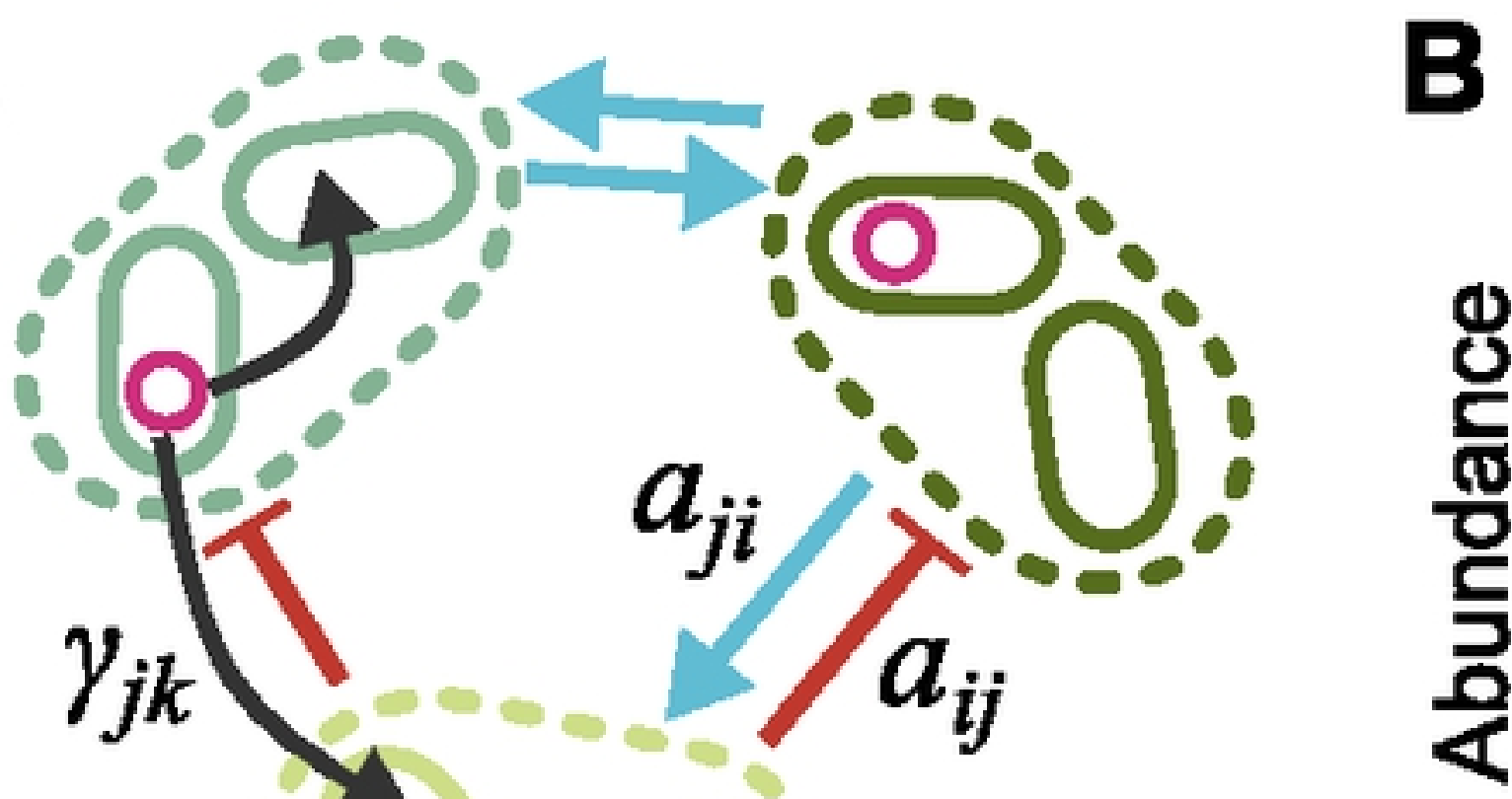

bioRxiv preprint doi: https://doi.org/10.1101/2022.02.25.481914; this version posted February 25, 2022. The copyright holder for this preprint (which was not certified by peer review) is the author/funder, who has granted bioRxiv a license to display the preprint in petpetuity. It is made vailable under aCC-BY 4.0 International license.

C

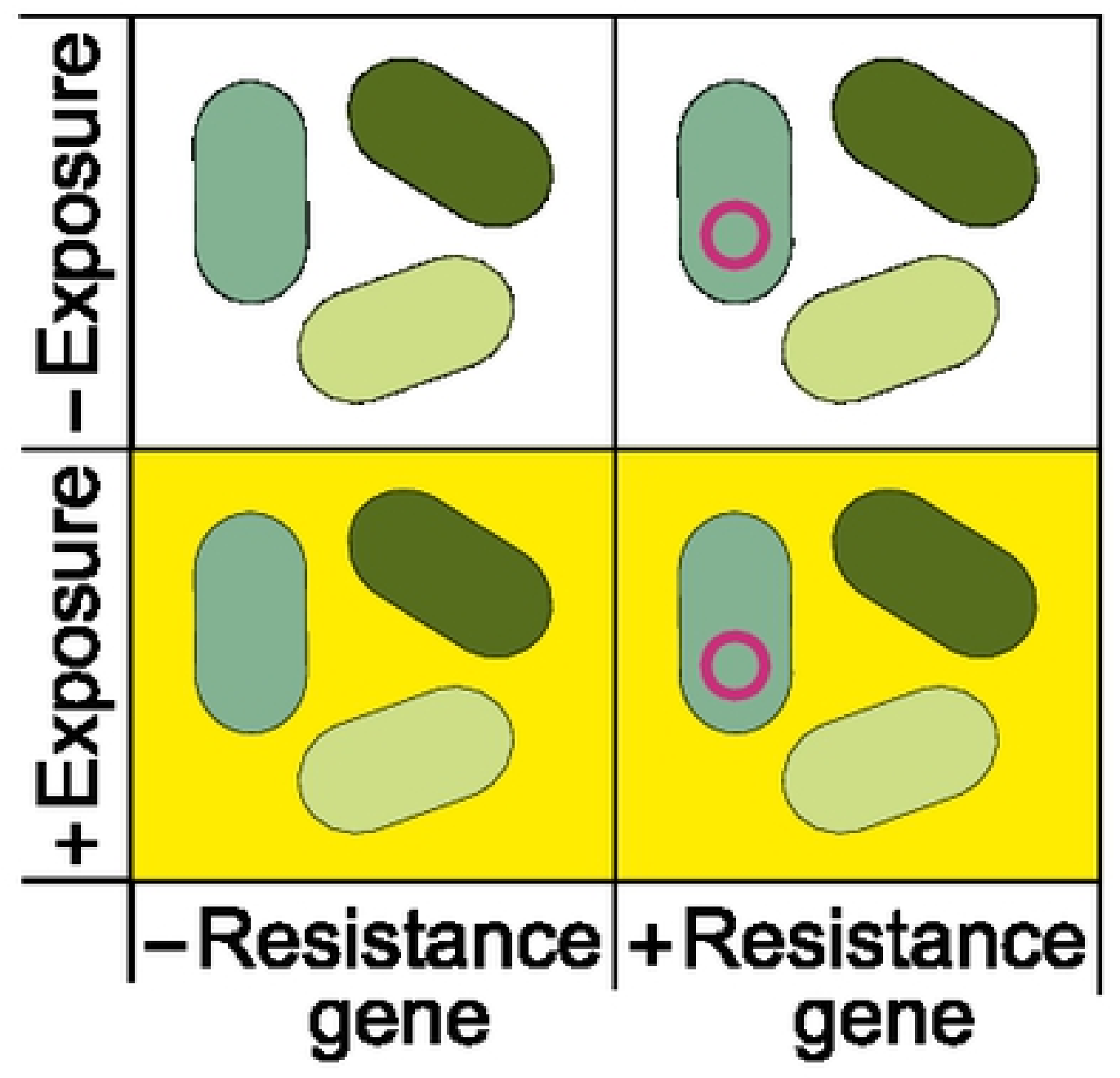

D

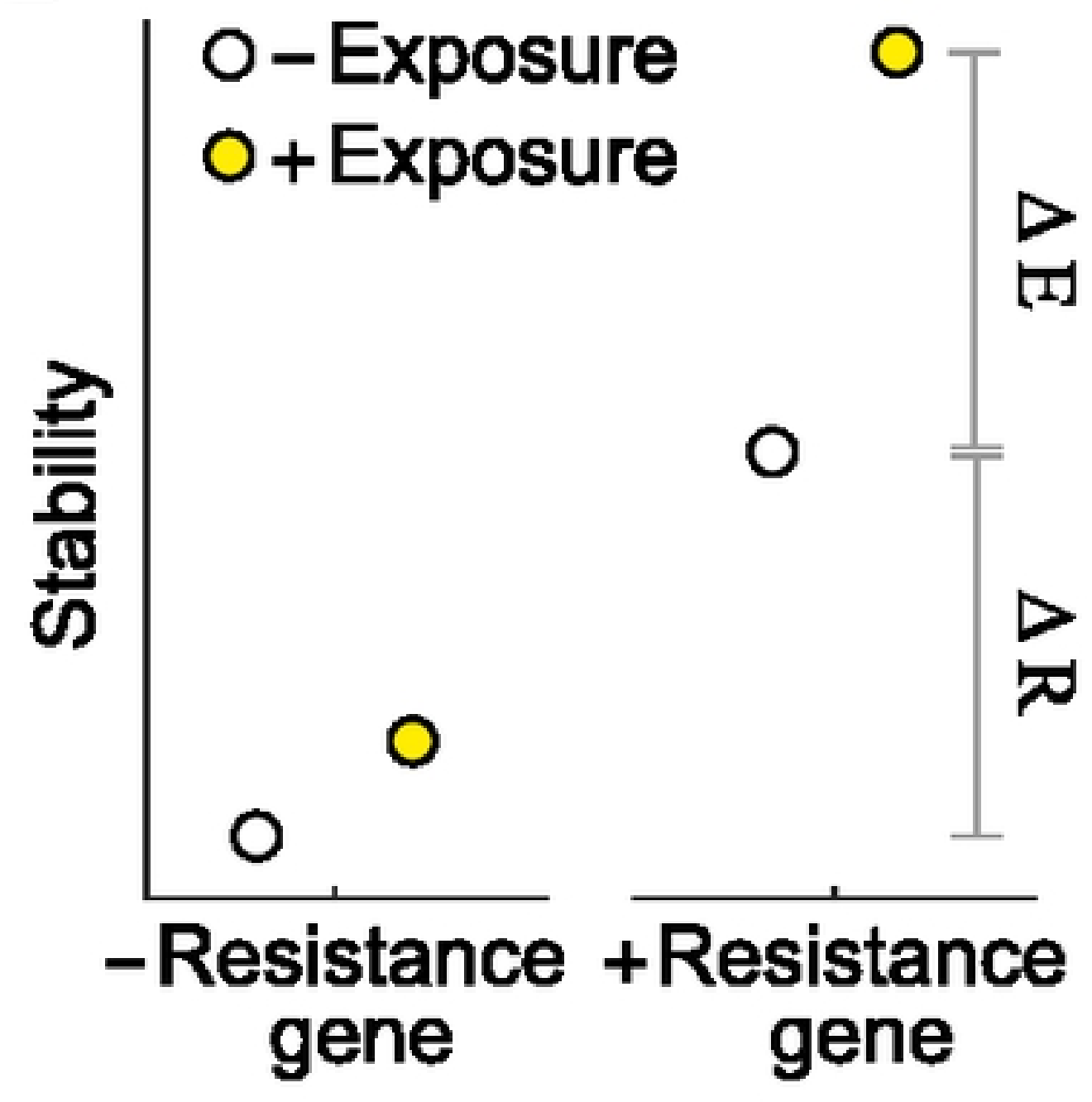

Total

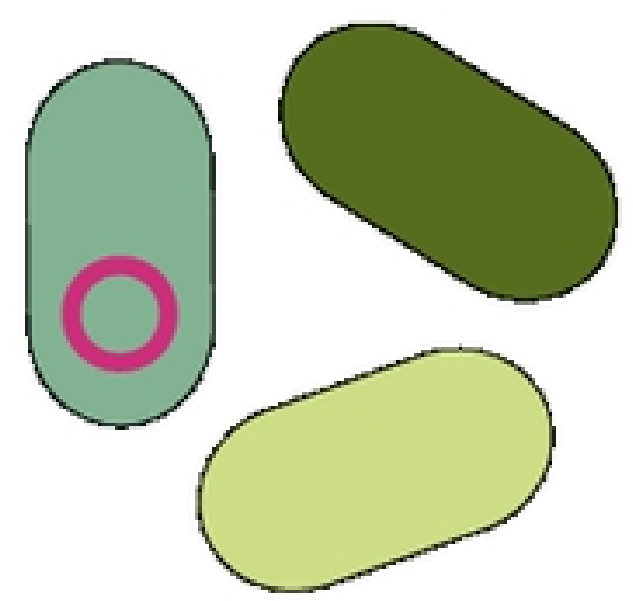

Background

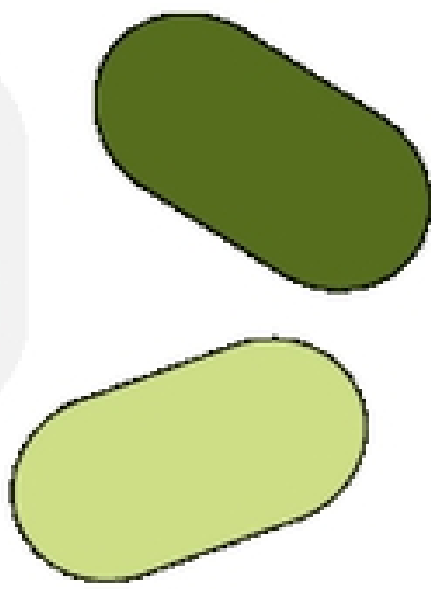

Focal

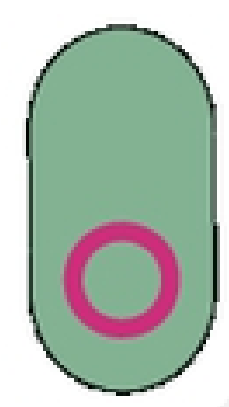

Figure 1 
